\title{
Magnetic Field Accuracy and Trim Coils in Wendelstein 7-X
}

\author{
J. Kißlinger*, T. Andreeva** \\ *Max-Planck-Institut für Plasmaphysik, EURATOM Ass., Boltzmannstr. 2, \\ D-85745 Garching, Germany \\ **Max-Planck-Institut für Plasmaphysik, Teilinstitut Greifswald, EURATOM Ass., \\ Wendelsteinstraße 1, D-17491 Greifswald, Germany
}

\begin{abstract}
The super-conducting magnet system of Wendelstein 7-X (W7-X) consists of five identical field periods (modules). Magnetic field errors arise if the modules are not exactly identical. Even small deviations in the coil shapes of the same type or misalignments of coils or modules break the periodicity of the system and cause error field components.

Simulation of the magnetic field perturbations which are expected has been done by the analysis of existing winding packages and statistical extrapolations of inaccuracies expected during assembly steps. Numerical experiment has shown that assembly errors should contribute significantly more than manufacturing errors of individual coils.

Compensation of the magnetic field perturbation can be done with help of the coil adjustment during the assembly or by the individual adjustment of all five modules. Further compensation of field errors is possible with additional coils. The existing control coils in W7-X can be used for error field compensation, however their efficacy is limited. Therefore, solutions employing normal-conducting trim coils outside the cryostat vessel are also considered here.
\end{abstract}

\section{Introduction}

The advanced stellarator Wendelstein 7-X (W7-X) /1,2/, has a magnetic field optimised for good plasma confinement and stability. The device, which is currently under construction at IPP in Greifswald, has the following main parameters: $\mathrm{R}=5.5 \mathrm{~m}, \mathrm{a}=0.52 \mathrm{~m}, \mathrm{~B}_{00}=3 \mathrm{~T}$. The modular super-conducting magnet system of W7-X is designed for stationary operation and consists of five identical field periods (modules). The inherent islands and separatrix at the plasma edge region are used for divertor operation.

Magnetic field errors arise if the modules are not exactly identical. Even small deviations in the coil shapes of the same type or misalignments of coils or modules break the periodicity 
of the system and cause error field components /3/. These components can be resonant in lowest order to the rotational transform, which is unity in most of the reference configurations. Such field perturbations lead to additional islands and cause asymmetric thermal loads on the divertor targets.

\section{Expected magnetic field accuracy}

At the end of July, 2005, 44 of the 50 non planar and all planar coil winding packages were manufactured. Their shapes show systematic deviations to the nominal geometry and statistical deviations from the mean filament geometry of all investigated winding packs of the same type. Only the statistical deviations, called shape errors, generate error field components with periodicity other than five. The systematic deviations only add negligible field components. Assuming that the remaining winding packs show statistical deviations in the same range, the dominant Fourier components of the error fields $\Delta B_{11} / B_{00}$ and $\Delta B_{22} / B_{00}$ would contribute $0.59 \cdot 10^{-4}$ and $0.62 \cdot 10^{-4}$, respectively, to the field errors. The error field $\Delta \mathrm{B}$ points perpendicular to the undisturbed magnetic field on a magnetic surface near the edge. Geometrical inaccuracies during assembly of the magnet system, called assembly errors, result from misalignments of single coils, distortions during welding of the lateral support elements and from misalignments of half-modules and modules. Numerical analyses have shown that statistical extrapolations of inaccuracies occurring during all assembly steps result in an average deviation of each coil of $3.5 \mathrm{~mm}$ and in Fourier components $\Delta \mathrm{B}_{11} / \mathrm{B}_{00}$ and $\Delta \mathrm{B}_{22} / \mathrm{B}_{00}$ of about $3 \cdot 10^{-4}$ and $1.5 \cdot 10^{-4}$, respectively. A detailed description of the expected assembly errors is given in /4/. Comparing the different values, it is evident that assembly errors contribute significantly more than manufacturing errors of individual coils.

\section{Magnetic field correction by coil adjustment}

A minimisation of the magnetic field perturbation introduced would be possible by ordering the non-planar coils in a toroidal sequence with the aim of best cancelling of the error field components. The result of such a minimisation is the optimal position of each coil. However, this procedure requires that the construction and tests of a distinct coil are completed when it is needed for assembly. No other coil of the same type could be used at its position.

A more practicable way for reducing the shape errors would be the adjustment of the exact coil position and alignment during the half module assembly. The geometrical data of each winding pack are measured at about 800 points. With a best fit procedure from this geometry 
data the co-ordinates of 8 reference points on the winding pack are defined. These reference points, called pins, carry the basic geometry information during all further machining and assembly steps. Introducing some small corrections in these pin co-ordinates, in the range of a few mm, it is possible to reduce the magnetic field errors. Such corrections consist of application of small shifts and tilts to the winding packs with respect to the reference design position; see also $/ 5 /$.

It is planned during the final assembly step of the magnet system of W7-X, to adjust each of the individual modules within a sphere of $5 \mathrm{~mm}$ radius at the position of the flanges of the support structure. This is done by applying shims between the module sectors. This correction measure is able to reduce shape and assembly error field components known at that time. This method is effective for the low order components $B_{11} / B_{00}$ and $B_{22} / B_{00}$ with amplitudes $<3 \cdot 10^{-4} / 5 /$.

\section{Compensation using trim coils}

In spite of the large effort made for the precise manufacturing and assembly of the W7-X coil system some significant uncertainties remain. Tolerances in machining and positioning of the coils, welding distortions and variations in material data can cause substantial field perturbations resonant to iota $=1$.

Additional compensation of field errors could be achieved by extra coils. In W7-X, there will be 10 control coils installed inside the vacuum vessel, foreseen to optimise the boundary magnetic configuration, but which can also be used for error field compensation. However, their capability with respect to the $B_{11}$ Fourier component is too low, only $0.4 \mathrm{mT}$ can be generated; see Table I. From this point of view an additional set of trim coils is required. To find an appropriate solution for trim coils some alternatives were investigated: normal conducting coils inside the plasma vessel and outside the cryostat vessel as well as superconducting helical windings inside the cryostat; for details see $/ 6 /$. The coils close to the plasma and the helical windings have the advantage that the required currents are smaller compared with those needed outside the cryostat vessel. However the technical realisation and the accessibility is easier in the case of normal conducting outer coils. For the generation of $\mathrm{B}_{11}$ and small $\mathrm{B}_{22}$ components it is sufficient to have five trim coils (one per field period) on the outer vessel. With respect to the numerous ports, two alternative positions have been identified: one on top of the outer cryostat vessel in the vicinity of the triangular plasma cross-section at toroidal angle $\varphi=40^{\circ}$ (type 1) /5/ and one centred on the radial outside at 
the bean shape plasma cross-section at $\varphi=0, Z=0$ (type 2); see Figs. 1 and 2. While the coil on top of the vessel has a saddle like 3-dimensional shape, the other one is designed as a plane coil. Both have dimensions of about $2 \mathrm{~m} \times 2 \mathrm{~m}$. Using one coil per field period, each coil should have a current capability of 100kA-turns.
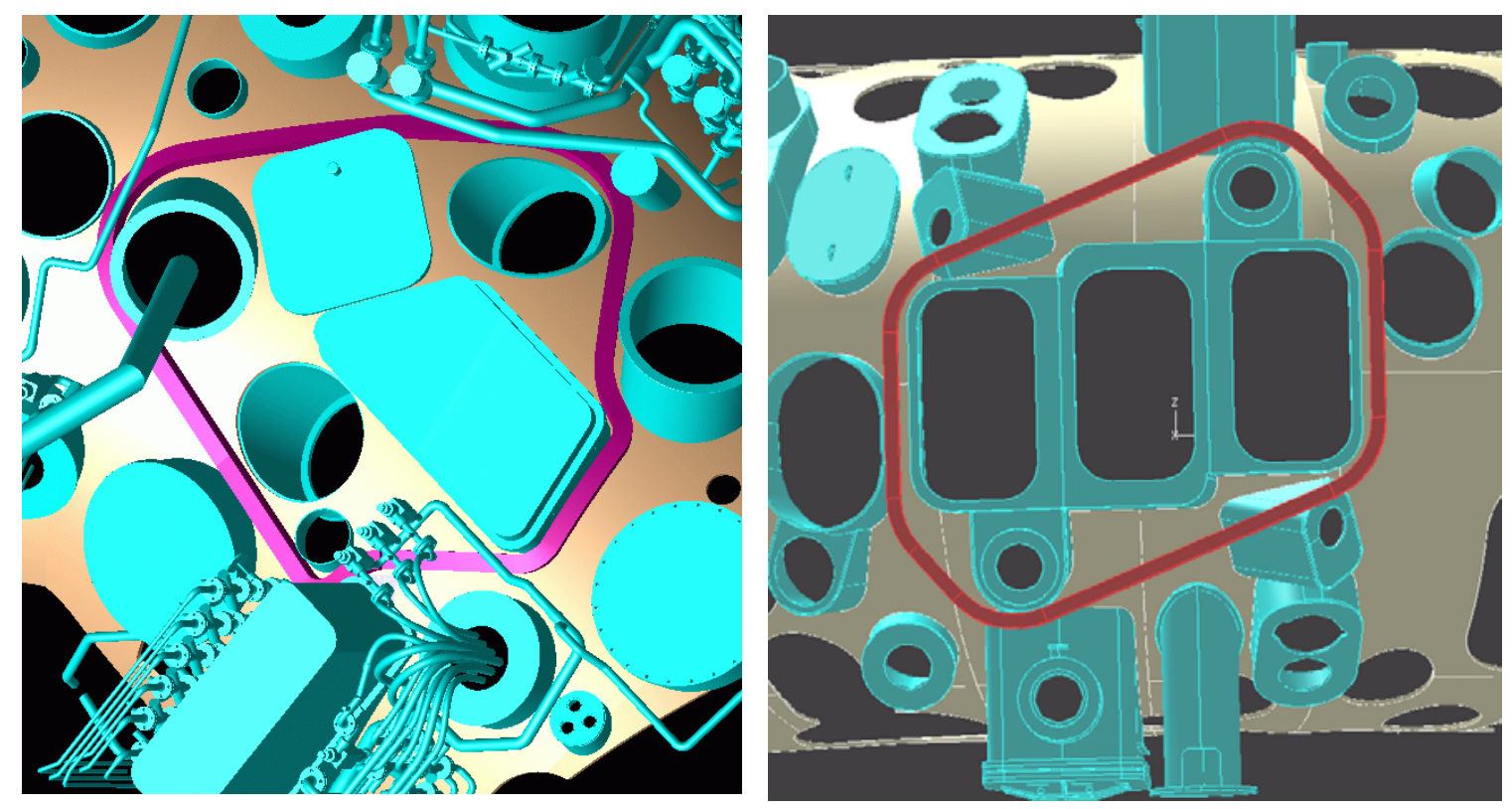

Fig. 1 CAD plot of the trim coil type 1 on top of the outer vessel (left) and the planar coil located at the radial outside in the vicinity of the large ports (right).

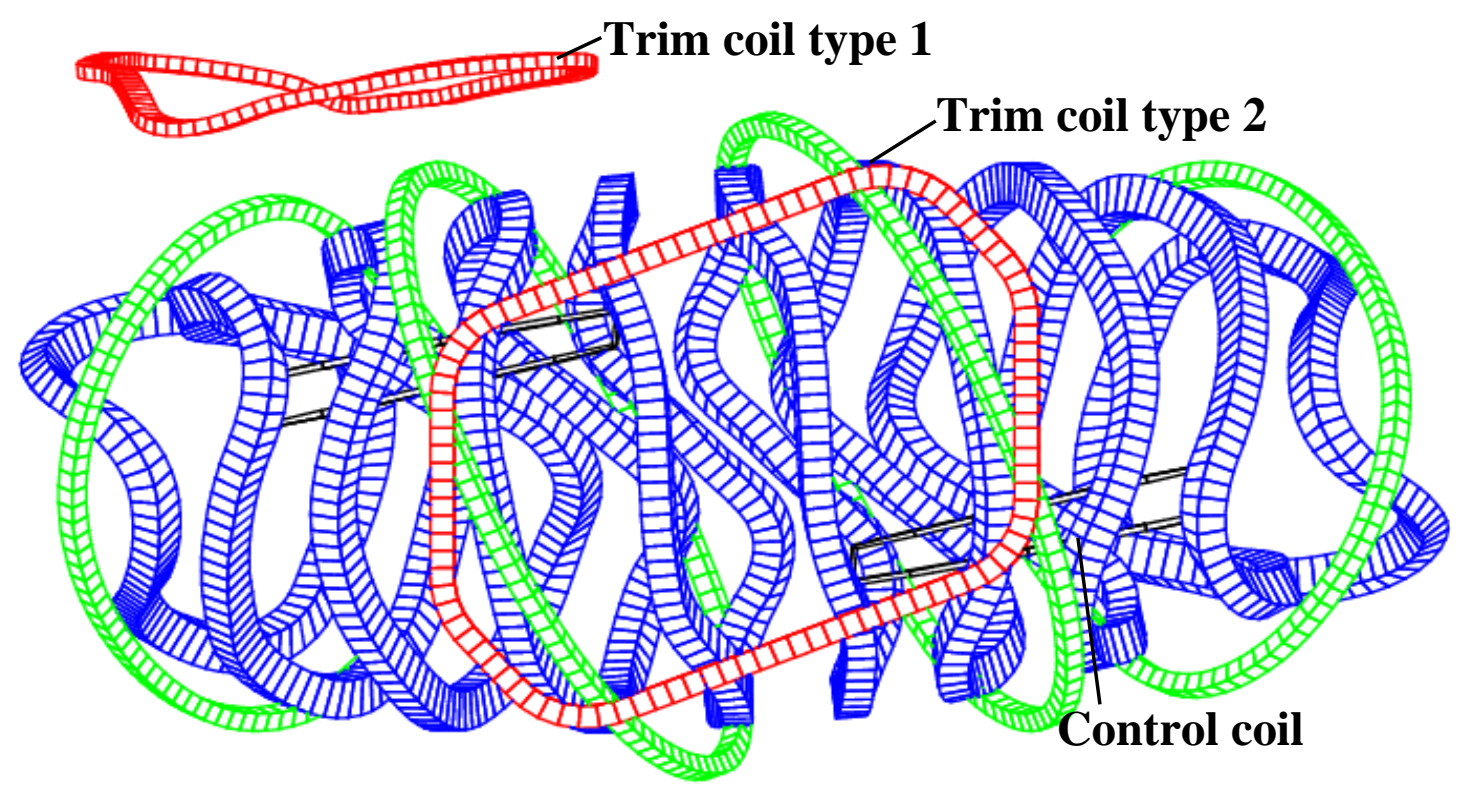

Fig. 2 Sketch of the coil system of Wendelstein 7-X (one period) including the control coils and the two options of trim coils. 
As an example, Fig. 3 shows the Poincarè plot of a perturbed field with error field harmonics of $\Delta \mathrm{B}_{11} / \mathrm{B}_{00}=1.66 \cdot 10^{-4}$ and $\Delta \mathrm{B}_{22} / \mathrm{B}_{00}=0.31 \cdot 10^{-4}$ and Fig. 4 the Poincarè plot of the corrected field using the planar coils. Since the error field has periodicity one, the currents in the trim coils also need this periodicity.
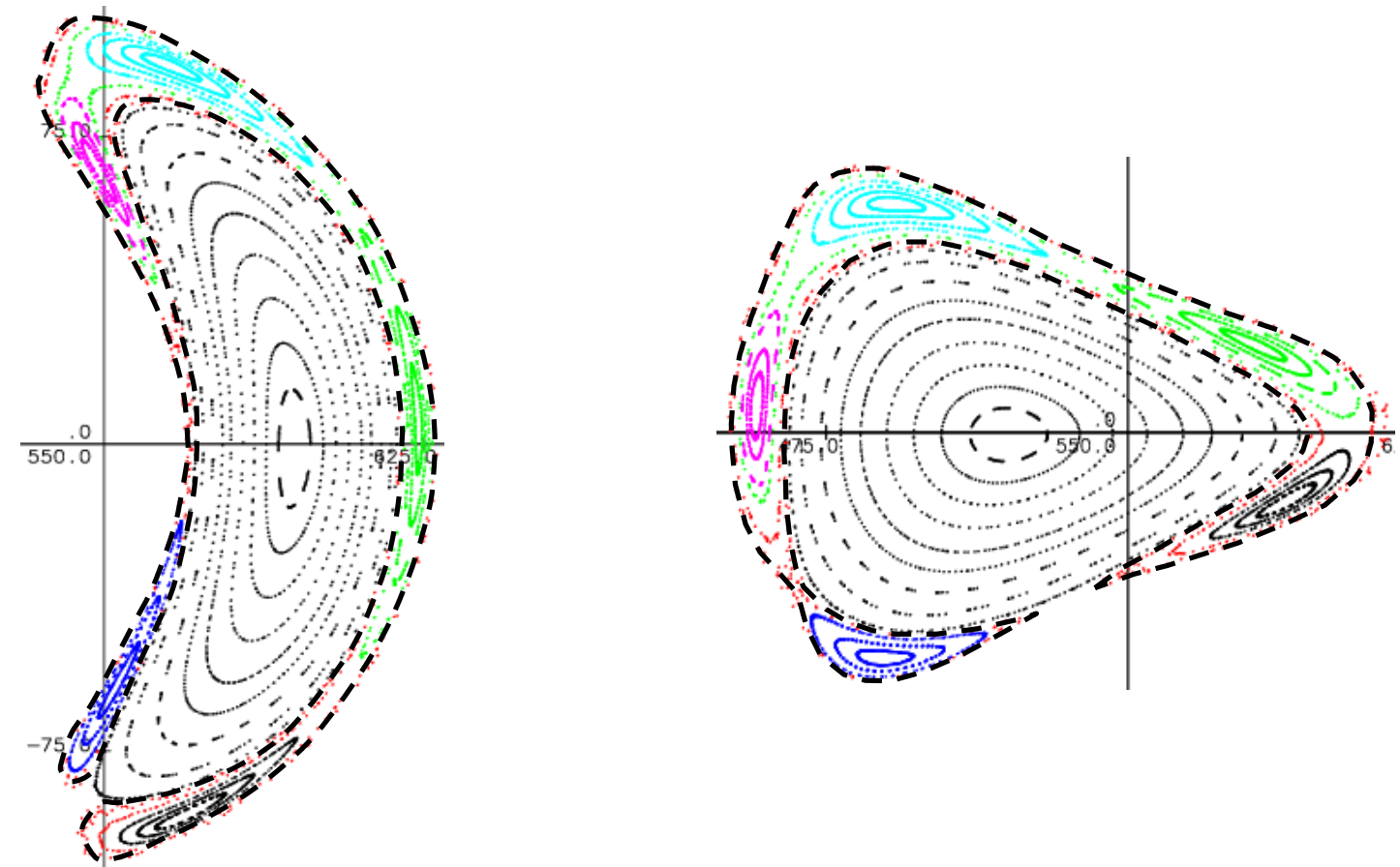

Fig. 3 Poincaré plot of a perturbed field of W7-X standard configuration. The error field is caused by statistical inclination of the non planar coils of up to $0.1^{\circ}$ with respect to the vertical and horizontal axis. The figure shows two poloidal plains at $\varphi=0^{\circ}$ (left part) and $\varphi=36^{\circ}$ (right part), which are up-down symmetric in the unperturbed case. In order to guide the eye, the 1/1 island, resulting from the error field and enclosing the remnants of the original 5/5 islands, is indicated by a dashed line.

Table I: Fourier components in $\mathrm{mT} / \mathrm{kA}$ generated by the control coil and the Trim coil type 1 and 2. In the case of only one trim coil per field period, $B_{33}$ and $B_{44}$ are side bands of $B_{22}$ and $\mathrm{B}_{11}$, respectively, and are not individually adjustable.

\begin{tabular}{|l|c|c|c|c|c|}
\hline \multicolumn{1}{|c|}{ Fourier component } & $\mathbf{B}_{\mathbf{1 1}}$ & $\mathbf{B}_{\mathbf{2 2}}$ & $\mathbf{B}_{\mathbf{3 3}}$ & $\mathbf{B}_{\mathbf{4 4}}$ & $\mathbf{B}_{\mathbf{5 5}}$ \\
\hline Control coil & 0.016 & 0.040 & 0.052 & 0.053 & 0.065 \\
\hline Trim coil type 1 & 0.016 & 0.006 & 0.001 & 0.001 & 0.001 \\
\hline Trim coil type 2 & 0.02 & 0.008 & 0.006 & 0.003 & 0.002 \\
\hline
\end{tabular}



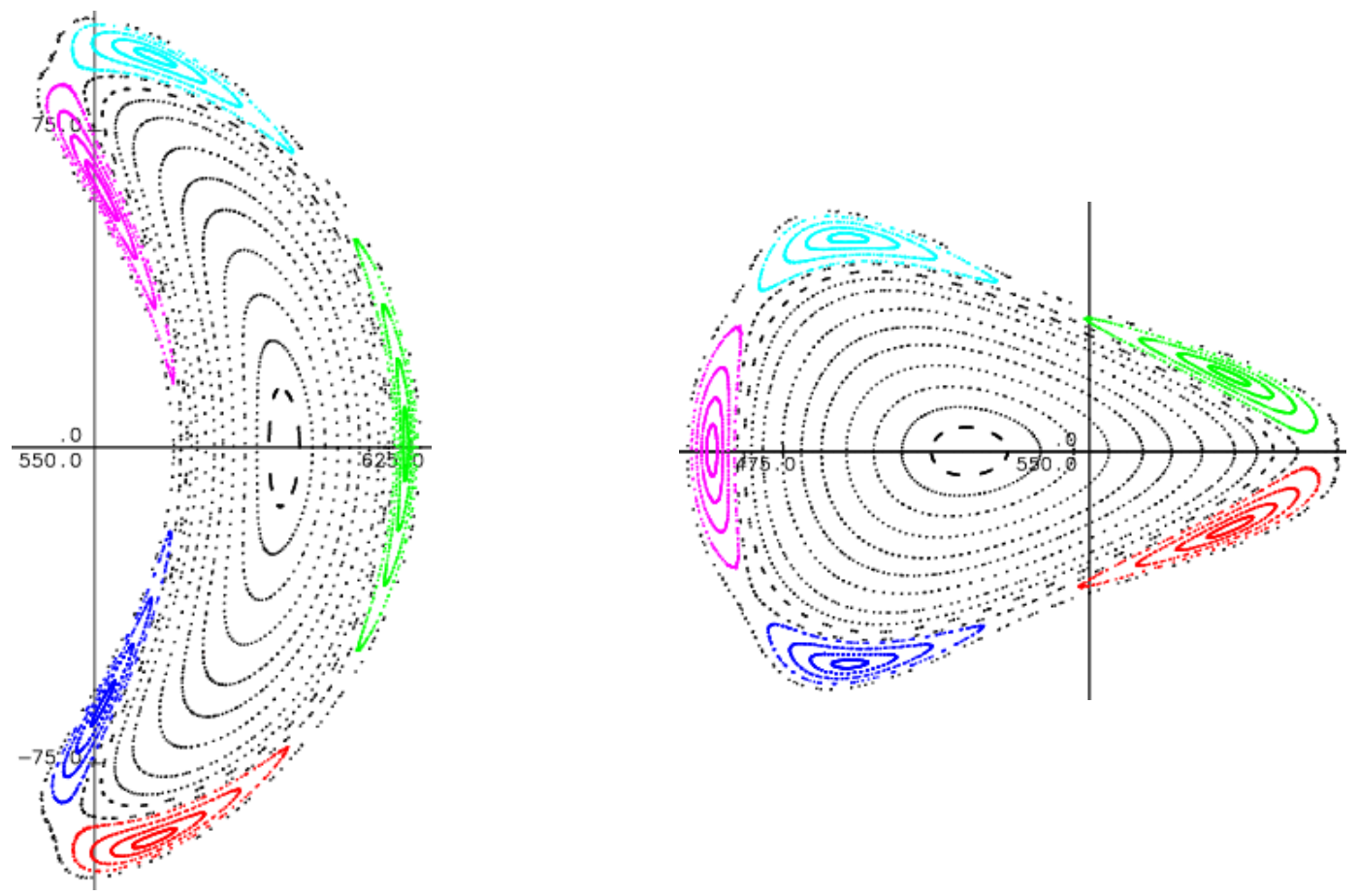

Fig. 4 Poincaré plot of corrected field of fig. 2 using trim coil type2. The currents used in coils in the modules 1 to 5 are $-12 k A,-29 k A, 9 k A, 22 k A$ and $2.5 k A$. The 1/1 island has vanished and the up-down symmetry is reconstructed. The symmetric 5/5 islands are desired and favourable for divertor action.

\section{Forces on the trim coils}

The trim coils are in the stray field region of the non-planar and planar coils of the superconducting magnet system. The stray field is mainly generated by the planar coils and varies with the currents in these coils used to adjust the magnetic configuration. The maximum force per unit length reaches locally $13 \mathrm{kN} / \mathrm{m}$ in the high iota case with trim coil currents of 100kA and a magnet field strength of 3T (coil type 2). Fig. 5 shows the force distribution along the coil periphery. The forces are mainly generated by the self field and point radially outward from the trim coil centre. The asymmetry is due to the stray field of the superconducting coils and a net force results between the trim coils and the magnet system. Since the stray field comes mainly from the planar coils the net force is maximal in the configurations with large currents in these coils, e.g. low-shear, high-iota case. In the highiota configuration a toroidal component of $14 \mathrm{kN}$ and a vertical component of $10 \mathrm{kN}$ result. Taking into account the forces of the trim coils in all five modules the net forces can either result in a horizontal force of up to $40 \mathrm{kN}$ or in a moment of up to $500 \mathrm{kNm}$ (with respect to 
the vertical axis of the device) depending on the current distribution in the coils. The net force must be balanced through the thermal isolation of the cryostat as in the case of the gravitational force.

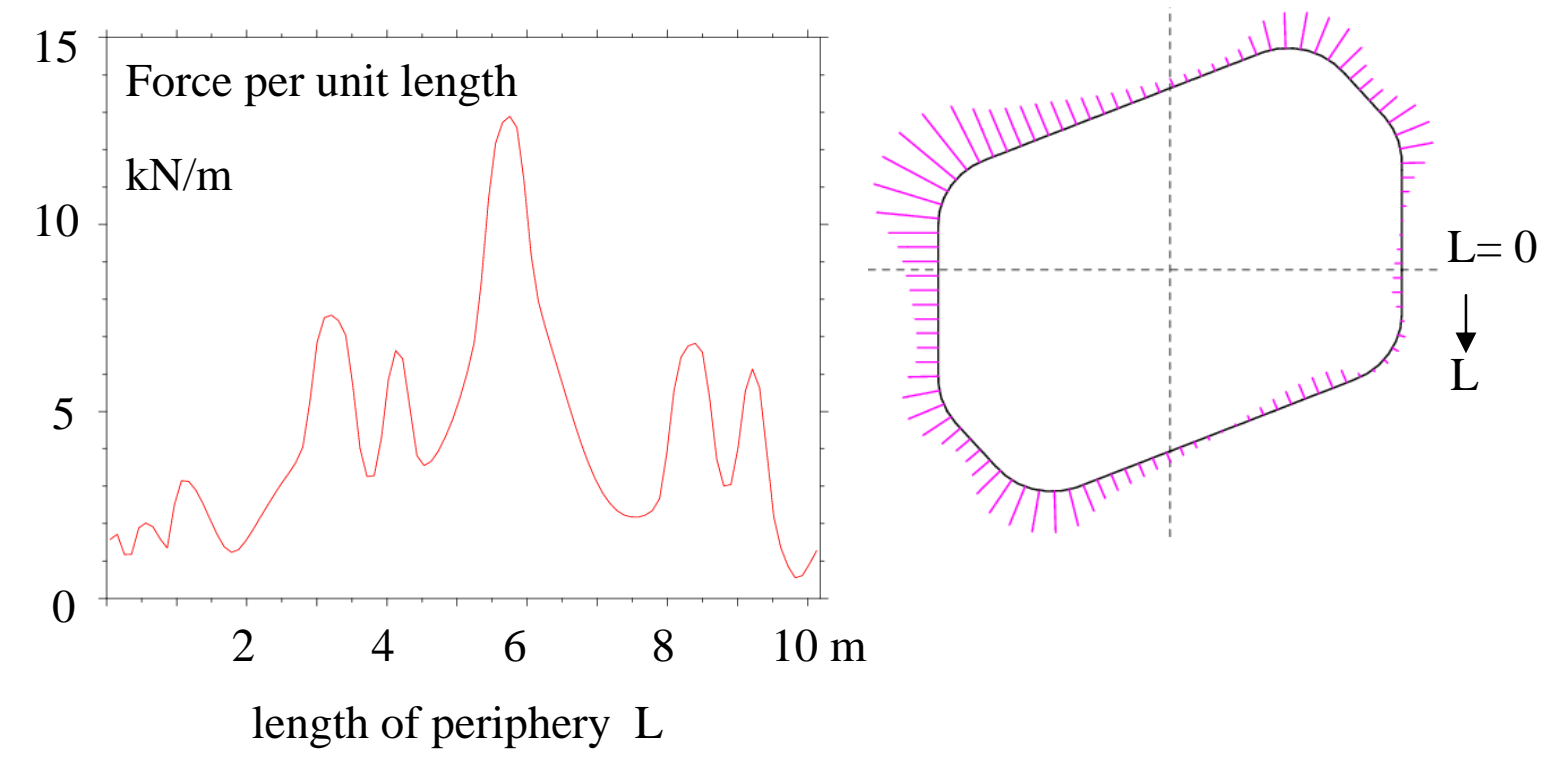

Fig.5 Distribution of the force per unit length along the coil periphery of the planar trim coils. Coil current 100kA, high- iota configuration

\section{Conclusions}

In W7-X, the intrinsic magnetic islands $5 / 5$ according to iota $=1$ will be used for divertor action. If the five fold symmetry of the device is not maintained, even very small deviations from the ideal symmetry of the five modules cause asymmetric loads on the divertor targets. This sensitivity requires a high geometrical repeating precision during manufacturing and assembly of the magnet system.

Most of the winding packs are already manufactured and their deviations cause field errors in the tolerable range. The expected assembly errors, however, will contribute significantly more to the field perturbation.

An individual adjustment of the five modules in the final assembly step offers the possibility to reduce low-order error field components known at that time.

The remaining uncertainties in the exact knowledge of the coil shape and position may require a further correction of the field. For this reason, positions and shapes of possible trim coils have been identified. With currents of $100 \mathrm{kA}$-turns in the trim coils $\Delta \mathrm{B}_{11} / \mathrm{B}_{00}$ of up to $5 \cdot 10^{-4}$ or $\Delta \mathrm{B}_{22} / \mathrm{B}_{00}$ of up to $2 \cdot 10^{-4}$ can be corrected. 
The electromagnetic forces acting on the trim coil are mainly generated by the self field and point radially outward from the trim coil centre. Due to the stray field from the superconducting coils an asymmetry is introduced and a net force between these coils and the trim coils exist and must be balanced through the thermal isolation.

\section{References}

/1/ C. Beidler et al., Physics and engineering design for Wendelstein 7-X, Fusion Technology, Vol. 17, 148-168, (1990)

/2/ Wanner, M., V. Erckmann, J.-H. Feist, et al, Status of WENDELSTEIN 7-X construction, Nuclear Fusion 43, 416-424 (2003)

/3/ F. M. Hamzeh, Formation and destruction of magnetic islands in toroidal systems, Physical Review Letters, 29, 1492-1497, (1972)

/4/ T. Andreeva, J.Kißlinger, Yu. Igitkhanov, Analysis of the magnetic field perturbations during the assembly of W7-X, Fusion Science and Technology, 46/2, 388-394, (2004)

/5/ J. Kißlinger, T. Andreeva, Correction possibilities of magnetic field errors in Wendelstein 7-X, Fusion Engineering and Design, 74 (2005) 623-626.

/6/ J. Kißlinger, Possibilities for Correction and compensation of magnetic field errors in Wendelstein 7-X, $14^{\text {th }}$ Stellarator workshop, Greifswald, Germany, Paper 014. 